\title{
VARIÁVEIS PLURI-DISCIPLINARES E TERMODINÂMICAS EM CONTEXTO COM ESTUDOS INTER- E TRANS- DISCIPLINARES
}

\author{
Paulo Pereira Martins Jr. \& Omar Campos Ferreira(*)
}

\begin{abstract}
This is the second paper in a series that intend to show the inter and transdisciplinary cartography. This view needs one especification of variable to be measured, studied and observed into Nature to answer the main question about structure and function of the ecosystems. Essential means answer the more fundamental questions about the morpho-functional condiction with the less cost. Here, will be showed in a short way the variables needed for this view. The biotic and abiotic variables are enphasized and the social-economic will be let for another study.
\end{abstract}

\section{INTRODUÇÃO}

Este é um segundo artigo de uma série com a qual se pretende apresentar as cartografias inter- e transdisciplinares. As abordagens disciplinar, pluri- , inter- e trans- disciplinares demandam uma especificação de variáveis a serem medidas, estudadas e observadas na Natureza de tal modo que em seu conjunto permitam responder às perguntas essenciais sobre estrutura e função dos ecossistemas. Essencial significa responder às questões mais fundamentais sobre as condições morfo-funcionais com o menor custo possível.

Todo estudo morfo-funcional deve por sua vez deve representar a geodinâmica própria de um sub-sistema natural, o que significa reconhecer os processos em atuação nas várias unidades sistêmicas. Por certo as diversas ciências especialistas estudam esses processos com diferentes enfoques em virtude do fato das perguntas serem dirigidas para questões específicas e especialistas. No entanto os estudos morfo-funcionais de ecossistemas e bacias hidrográficas devem responder às perguntas que dizem respeito às realidades intrínsecas desses sistemas, com ou sem a presença do homem. A própria presença do homem pode apenas ser abstraída em um primeiro tempo. Isto quer dizer que as variáveis derivadas da presença humana devem ser incorporadas na economia do sistema, esteja ele em equilíbrio ou em desintegração.

Diferente coisa é perguntar-se sobre os efeitos futuros de uma intervenção e o trato que se deve dar à presença humana como parte de fato do sistema. Podese considerar, e assim se considera neste estudo, que a própria presença humana pode ser um pouco ignorada em uma primeira etapa. Essa abstração deve ser considerada como um exercício para se apreender as relações estruturais e funcionais naturais como estejam. Por vezes a atuação do homem é tão marcante que desde o início dos estudos deve ser amplamente considerada.

Nesse estudo apresenta-se de modo sucinto as variáveis consideradas indispensáveis para as abordagens inter-e trans- disciplinares de uma região. Dá-se ênfase às variáveis abióticas e bióticas, deixando-se as variáveis sócio-econômicas para outro estudo.

\section{AS VARIÁVEIS PLURIDISCIPLINARES PARA OS ZONEAMENTOS}

A tabela 1 indica as variáveis cuja importância é inequívoca para ambas as abordagens, apresentando um esquema de agregação lógica por contexto e por aplicação. Outras variáveis poderão ser utilizadas com igual força de interesse, dependendo do tipo de questão que deva ser avaliada em dada região. A julgar para cada caso.

O zoneamento integrado poderá ser feito com o conjunto total de variáveis ou poderá ser feito com a associação cartográfica dos resultados de cada zoneamento segundo metodologia própria, conveniência e critérios a serem ajuizados por avaliação qualitativa e matemática.

\section{AS VARIÁVEIS PARA OSZONEAMENTO}

Dado que o recorte de terras homogêneas é distinto para eco-unidades e para os geo-hidrossistemas (Tabela 1) recomenda-se, conforme haja intenção de integrar os dois enfoques de pesquisa, a se adotar de modo diferenciado a nomenclatura de Moss (1985) para as diversas escalas de representação, a saber:

- as informações descritivas e os critérios vão ser distintos de escala para escala, envolvendo sejam os processos ecológicos, os termodinâmicos, os processos telúricos, os processos hídricos e os processos climáticos. Tudo isto implica em se identificar critérios rigorosos para as diversas situações, sem negligenciar as variáveis sensíveis para os tipos de impactos específicos a serem estudados e controlados. A escolha da escala contempla o grau de acurácia com que alguma decisão gerencial deverá ser tomada na gestão ambiental, no enquadramento de cursos d'água, na efetivação do enquadramento e no gerenciamento de uma bacia hidrográfica. 


\begin{tabular}{|c|c|c|c|c|c|c|c|c|c|}
\hline Contexto & Variáveis & $\begin{array}{l}\text { Morfo- } \\
\text { metria }\end{array}$ & $\begin{array}{c}\text { Capa- } \\
\text { cidade } \\
\text { assi- } \\
\text { mila- } \\
\text { tiva }\end{array}$ & $\begin{array}{l}\text { Quant. } \\
\text { /Qual. } \\
\text { águas }\end{array}$ & $\begin{array}{l}\text { Eco- } \\
\text { unida- } \\
\text { des }\end{array}$ & $\begin{array}{l}\text { Geo- } \\
\text { técni- } \\
\text { cas }\end{array}$ & $\begin{array}{l}\text { Eco- } \\
\text { silvi- } \\
\text { agrí- } \\
\text { cola }\end{array}$ & $\begin{array}{l}\text { Geo- } \\
\text { siste- } \\
\text { mas }\end{array}$ & $\begin{array}{l}\text { An- } \\
\text { trópi- } \\
\text { co }\end{array}$ \\
\hline \multirow{14}{*}{ Métrica } & Áreas das sub-bacias & $x$ & $x$ & $x$ & $x$ & $\mathrm{x}$ & $x$ & $x$ & $x$ \\
\hline & Comprimento Axial das sub-bacias & $x$ & $x$ & $x$ & & $x$ & & $x$ & \\
\hline & Fator de forma & $x$ & $x$ & $x$ & $\mathrm{x}$ & $x$ & & $x$ & \\
\hline & Coeficiente de compacidade & $x$ & $x$ & $x$ & $x$ & $x$ & $x$ & $x$ & \\
\hline & Altitude máxima & $x$ & $x$ & & & $x$ & $x$ & & \\
\hline & Altitude média & $x$ & $x$ & & & $x$ & & & \\
\hline & Altitude mínima & $x$ & $x$ & & $\mathrm{x}$ & $x$ & & & \\
\hline & Índice de diversidade do terreno & $x$ & $x$ & & $\mathrm{x}$ & $x$ & & $x$ & \\
\hline & Índice morfolitográfico & & $x$ & & $x$ & $x$ & & $x$ & \\
\hline & İndice pedogeomórfico & & & & & $x$ & & $x$ & \\
\hline & Direções angulares de drenagem & $x$ & $x$ & & & $x$ & & $x$ & \\
\hline & Índice de rugosidade de superfície & $x$ & & & $x$ & $x$ & & $x$ & \\
\hline & Áreas de litossomas & $x$ & & $x$ & & $x$ & $x$ & $x$ & \\
\hline & Áreas de morfotemas & $x$ & $x$ & $\mathrm{x}$ & $x$ & $x$ & $x$ & $x$ & \\
\hline \multirow{12}{*}{ Solos } & Áreas de tipos de solos & & $x$ & $x$ & $x$ & $x$ & $x$ & & $x$ \\
\hline & Drenagem de solos & & $x$ & $x$ & $x$ & $x$ & $x$ & & $x$ \\
\hline & Textura dos solos & & $\mathrm{x}$ & $x$ & $x$ & $x$ & $x$ & & $x$ \\
\hline & Porosidade & & & & & $x$ & & & \\
\hline & Índice de vazios & & & & & $x$ & & & \\
\hline & Teor de umidade & & & & & $x$ & & & \\
\hline & Massa específica natural & & & & & $x$ & & & \\
\hline & Grau de saturação & & & & & $x$ & & & \\
\hline & Limite de plasticidade & & & & & $x$ & & & \\
\hline & Índice de plasticidade & & & & & $x$ & & & \\
\hline & Coesão & & & & & $x$ & & & \\
\hline & Ângulo de atrito & & & & & $x$ & & & \\
\hline \multirow{7}{*}{ Hidrologia } & N. ${ }^{\circ}$ drenos por sub-bacia & & $x$ & $x$ & & & & $x$ & $x$ \\
\hline & Taxa de bifurcação de drenagem. & & $x$ & $x$ & & & & $x$ & $x$ \\
\hline & Densidade de drenagem & & $x$ & $x$ & & $x$ & $x$ & & $x$ \\
\hline & $\begin{array}{l}\text { Taxa bifurcação dren./ densidade de } \\
\text { drenagem }\end{array}$ & & $x$ & $\bar{x}$ & & & & $x$ & \\
\hline & $\begin{array}{l}\text { Declividade talvegue principal } \\
\text { máx/méd/mí }\end{array}$ & & $x$ & $x$ & $\mathrm{x}$ & $x$ & $x$ & $x$ & $x$ \\
\hline & Comp. total das drenagens & & $x$ & $x$ & & & & & \\
\hline & Vazões específicas & & $x$ & & & $x$ & $x$ & $x$ & $\mathrm{x}$ \\
\hline \multirow{11}{*}{$\begin{array}{c}\text { Clima } \\
\text { E } \\
\text { Trocas } \\
\text { De } \\
\text { Energia } \\
\text { E } \\
\text { Massa }\end{array}$} & Evapotranspiração potencial & & $x$ & $x$ & $x$ & $x$ & $x$ & & \\
\hline & Evapotranspiração real & & $x$ & $x$ & $x$ & $x$ & $x$ & & \\
\hline & Precipitação anual real & & $x$ & $x$ & $x$ & $x$ & $x$ & & \\
\hline & $\begin{array}{l}\text { Medida anual de decomposição do } \\
\text { folhedo como \% da produção de } \\
\text { húmus }\end{array}$ & & & & $x$ & & $x$ & & \\
\hline & $\begin{array}{l}\text { Produtividade primária nítida, valor } \\
\text { não ajustado }\end{array}$ & & & & $x$ & & $x$ & & \\
\hline & $\begin{array}{l}\text { Produtividade primária potencial } \\
\text { nítida, valor não ajustado }\end{array}$ & & & & $x$ & & $\mathrm{x}$ & & \\
\hline & İndice de performance do solo & & & & $x$ & & $x$ & & \\
\hline & $\begin{array}{l}\text { Produtividade primária nítida } \\
\text { potencial média }\end{array}$ & & $x$ & & $\mathrm{x}$ & & $\mathrm{x}$ & & \\
\hline & Produtividade primária acima do chão & & $x$ & & $x$ & & $x$ & & $x$ \\
\hline & Escoamento superficial acumulado & & $x$ & & $x$ & & $x$ & & \\
\hline & Capacidade acumulação & & $x$ & $x$ & $x$ & & $x$ & & \\
\hline \multirow{4}{*}{$\begin{array}{l}\text { Irriga- } \\
\text { ção }\end{array}$} & SAR taxa de absorção de $\mathrm{Na}$ & & & & & & $\mathrm{x}$ & & \\
\hline & $\mathrm{CSR} \mathrm{NaCO}_{3}$ residual & & & & & $x$ & $x$ & & $x$ \\
\hline & Índice de saturação de Langelier & & & & & $x$ & $\mathrm{x}$ & & $\mathrm{x}$ \\
\hline & Índice de Larson & & & & & $\mathrm{x}$ & $\mathrm{x}$ & & $\mathrm{x}$ \\
\hline
\end{tabular}




\begin{tabular}{|c|c|c|c|c|c|c|c|c|c|}
\hline Contexto & Variáveis & $\begin{array}{l}\text { Morfo } \\
\text { metria }\end{array}$ & $\begin{array}{c}\text { Capa- } \\
\text { cidade } \\
\text { assi- } \\
\text { mila- } \\
\text { tiva }\end{array}$ & $\begin{array}{l}\text { Quant } \\
\text { /Qual } \\
\text { águas }\end{array}$ & $\begin{array}{c}\text { Eco- } \\
\text { unida- } \\
\text { des }\end{array}$ & $\begin{array}{l}\text { Geo- } \\
\text { técni- } \\
\text { cas }\end{array}$ & $\begin{array}{l}\text { Eco- } \\
\text { silvi- } \\
\text { agrí- } \\
\text { cola }\end{array}$ & $\begin{array}{c}\text { Geo- } \\
\text { siste- } \\
\text { mas }\end{array}$ & $\begin{array}{l}\text { An- } \\
\text { trópi- } \\
\text { co }\end{array}$ \\
\hline \multirow{10}{*}{$\begin{array}{c}\text { Geologia } \\
\text { Aplicada } \\
e \\
\text { Geo- } \\
\text { tecnia }\end{array}$} & Nível freático & & & & $x$ & $x$ & & & $x$ \\
\hline & Topologia de fraturas & $x$ & $x$ & $x$ & $x$ & $x$ & $x$ & $x$ & $x$ \\
\hline & Zonas preferenciais de recarga & & $x$ & $x$ & $x$ & $x$ & $x$ & $x$ & $\mathrm{x}$ \\
\hline & Área Real de erosão & & $x$ & $x$ & $x$ & $x$ & $x$ & $x$ & $x$ \\
\hline & Área potencial de erosão & & $x$ & $x$ & $x$ & $x$ & $x$ & $x$ & $x$ \\
\hline & Área de e para empréstimos & & & $x$ & $x$ & $x$ & $x$ & & $x$ \\
\hline & Áreas de mineração & & & $x$ & & $x$ & & & $x$ \\
\hline & Áreas de prospectos minerais & & $x$ & & & $x$ & & & $x$ \\
\hline & Vulnerabilidade a fundações & & & & & $x$ & $\mathrm{x}$ & $x$ & $\mathrm{x}$ \\
\hline & Áreas geo-vulneráveis & & & & & $x$ & & & \\
\hline \multirow{20}{*}{ Antrópico } & Vias & & & $x$ & & $x$ & & $x$ & $x$ \\
\hline & Áreas urbanas & & $x$ & $x$ & & $x$ & & & $x$ \\
\hline & Indústrias & & $x$ & $x$ & & $x$ & & & $x$ \\
\hline & Silvicultura & & & & & $x$ & $x$ & & $x$ \\
\hline & Portos & & & $x$ & & $x$ & & $x$ & $x$ \\
\hline & Canais fluviais & & & $x$ & & $x$ & $x$ & $x$ & $x$ \\
\hline & Molhes e marinas & & & $x$ & & $x$ & & $x$ & $x$ \\
\hline & Diques & & $x$ & & & $x$ & & & $x$ \\
\hline & Barragens & & $x$ & $x$ & & $x$ & & & $x$ \\
\hline & Fontes de poluição sólida & & $x$ & $x$ & & $x$ & $x$ & & $x$ \\
\hline & Fontes de poluição líquida & & $x$ & $x$ & & & $x$ & & $x$ \\
\hline & Fontes de poluição gasosa & & & & & & $x$ & & $x$ \\
\hline & Desmatamento & & $x$ & $x$ & $x$ & & $x$ & & $x$ \\
\hline & Conurbação & & $x$ & $x$ & & $x$ & $x$ & & $x$ \\
\hline & Hidro-elétricas & & & $x$ & & $x$ & & & $x$ \\
\hline & Centrais nucleares & & $x$ & $x$ & & $x$ & & & $x$ \\
\hline & Carvoejamento & & $x$ & $x$ & $x$ & $x$ & $x$ & & $x$ \\
\hline & Parques de reservas & & & & $x$ & & $x$ & & $x$ \\
\hline & Espaço agrícola & & $x$ & $x$ & $x$ & $x$ & $x$ & & $x$ \\
\hline & Efluentes e dispersão & & $x$ & $x$ & & $x$ & $x$ & & $x$ \\
\hline \multirow{8}{*}{$\begin{array}{c}\text { Hidro- } \\
\text { geo- } \\
\text { químico }\end{array}$} & $\mathrm{pH}$ & & $x$ & $x$ & & & & & \\
\hline & Eh & & $x$ & $x$ & & & & & \\
\hline & Temperatura & & $x$ & $x$ & & & $x$ & & \\
\hline & Condutividade elétrica & & $x$ & $x$ & & & & & \\
\hline & Oxigênio dissolvido & & $x$ & $x$ & & & & & \\
\hline & Demanda bioquímica $\mathrm{O}_{2}$ & & $x$ & $x$ & & & & & \\
\hline & Demanda química $\mathrm{O}_{2}$ & & $x$ & $x$ & & & & & \\
\hline & Colifecais & & $x$ & $x$ & & & & & \\
\hline
\end{tabular}

Tabela 1: Variáveis mais significativas para estudos morfo-funcionais de ecossistemas e bacias hidrográficas a nível regional.

Table 1: More significant variables for morpho-funcional studies of ecosystems and hidrographic basin in a regional approach. 


\section{ALGORÍTMOS, QUANTIFICAÇÃOE RESULTADOS}

A quantificação se dá em diversos níveis a partir de:

(1) disciplinar,

(2) ao pluridisciplinar,

(3) ao interdisciplinar e

(4) ao transdisciplinar.

Defini-se o disciplinar como a quantificação própria e específica a qualquer disciplina; o pluridisciplinar ocorre quando dos mapeamentos temáticos conseguese estabelecer por relação de proximidade fenomenológica uma quantificação, visando atender questões específicas como, por exemplo, o uso agrícola e o controle da quantidade da água; o interdisciplinar ocorre quando todos os domínios fenomenológicos são tratados concomitantemente a partir de suas variáveis de modo a se estabelecer as relações de hierarquia, importância relativa, sinergia, próprias para as modelagens termodinâmica e sistêmica. $\mathrm{O}$ transdisciplinar tem como exemplo notável a análise exergética que permite que uma disciplina como a Termodinâmica seja usada de modo transdisciplinar para descrever fenômenos em diversos domínios sistêmicos distintos e também as relações entre os mesmos.

$\mathrm{O}$ tratamento quantificado deve atender às seguintes premissas:

(1) todos os subconjuntos de (i) a (vii) devem ser definidos com significativa intersecção;

(2) classificar o território em unidades homogêneas por subconjunto de (i) a (vii);

(3) determinar a hierarquia e a sinergia entre as variáveis classificadoras;

(4) estabelecer matrizes de correlação de importância relativa das variáveis;

(5) determinar os vários critérios de sensibilidade dos subsistemas;

(6) estabelecer os vários critérios de sustentabilidade em função de semelhanças, anisotropia, vulnerabilidade, processos geodinâmicos predominantes e a biodiversidade;

(7) descrever as trocas de energia, massa e informação com as variáveis exergia e emergia (Odum, 1996) para permitir apresentar com clareza as relações quantificadas entre diferentes sistemas.

Para se realizar a tarefa própria à Abordagem Interdisciplinar (Martins Jr., 1997) derivam-se as variáveis a partir das diversas disciplinas especialistas, dos mapeamentos temáticos e pluridisciplinares, que em seguida serão tratadas primeiramente pela análise hierárquica ascendente e pelas análises fatoriais aplicadas às áreas homogêneas detectadas. Aplica-se as análises discriminantes para resolver-se as discriminações finas ainda pendentes. Análises de séries temporais podem ser necessárias seja para sistemas e processos em regime permanente e/ou catastrófico.

Tais análises produzem:
(1) a agregação das sub-bacias ou das unidades de malha no terreno em áreas homogêneas para os subconjuntos (i) a (vii);

(2) identifica as variáveis com maior peso no vetor fator, logo com maior significância relativa;

(3) as pendências na identificação/ diferenciação entre áreas homogêneas e/ou conjunto de variáveis. A partir de então a análise se dará com a busca de relações entre duas, três e/ou quatro variáveis concomitantemente tal que sejam expressas como leis de relações complexas inter subsistemas, que de modo geral não seriam vistas de modo pluridisciplinar.

Tais produtos são totalmente distintos daqueles estritamente disciplinares e pluridisciplinares, porque são expressos no campo de relações sistêmicas e organizados em hierarquias e homeoarquias.

Com a Abordagem Interdisciplinar é possível estudar-se as diferenças entre as várias classificações de modo a se poder apreender como os diversos subconjuntos das variáveis geotécnicas, dos geo-sistemas, da morfometria, das trocas de energia/massa/ informação, da quantidade/qualidade das águas, da capacidade assimilativa de cursos d'água permitem classificar os sistemas naturais de tal modo a se observar e descrever como estes sub-conjuntos estruturais e/ou fenomenológicos se imbricam nas bacias hidrográficas e ecossistemas regionais.

Os estudos de classificação em áreas homogêneas se fazem com o critério rigoroso de intersecção entre todos os subconjuntos de dados tal que se possa obter até oito cartas definitivas que caracterizem a região e permitam descrever as relações sistêmicas.

Essas fornecem as:

(a) zonas homogêneas;

(b) a hierarquia e sinergia entre variáveis e

(c) as leis mais abrangentes que descrevem relações sistêmicas tanto estruturais quanto referentes a processos.

Esse conjunto de informações regionais deverá ser decodificado para aspectos locais em cima de perguntas específicas para questões também específicas.

Essas determinações podem prover os recursos para uma modelagem geo-ecológica dos sistemas naturais e culturais existentes. As modelagens devem atender aos critérios de prever:

(1) a adequação para projetos de silvicultura e agrícolas com coerência sistêmica;

(2) oferecer um quadro para estudos referentes aos assentamentos humanos e segurança dos geo-recursos;

(3) permitir determinar modelos de capacidade assimilativa dos cursos d'água, visando as atividades de controle/licenciamento/monitoramento;

(4) definir os ecossistemas pelas trocas de energia/ massa/informação tal que as condições dessas trocas sejam as características normativas para políticas de preservação/conservação/intervenção;

(5) definir os geo-sistemas e a morfometria para os estudos hidrológicos/geológicos/de recursos naturais renováveis ou não; 
(6) modelar as atividades de controle de uso funcional dos recursos hídricos e

(7) estabelecer as zonas e tipologia de impactos para serem considerados à luz dos estudos sistêmicos.

\section{AS VARIÁVEIS DOS ESTUDOS TRANSDISCIPLINARES}

Os estudos transdisciplinares estão baseados no fato simples de que se possa ver um fenômeno a partir de [trans] alguma [outra disciplina] que não seja específica para a descrição do dito fenômeno. Um exemplo bem claro disto está na aplicação da Termodinâmica a outras ciências. Em particular assinalase o uso das variáveis exergia e emergia. (GeorgescuRoegen,1976); (Jörgensen, 1992); (Ayres et al., 1996). A transdiciplinaridade com bases na Termodinâmica é o único modo de se poder tratar coisas distintas com as mesmas variáveis e assim se obter uma verdadeira possibilidade de comparação. É o único modo com o qual se pode considerar o trabalho, a ordem, a desordem e a entropia dos processos e sistemas naturais.

Define-se a variável exergia como a quantidade de energia disponível em qualquer sistema, para realizar qualquer tipo de trabalho, ou para se degradar, quando uma parte ou a totalidade do sistema entra em declínio (retorno à máxima entropia). A exergia aponta para o futuro do sistema, porque indica a condição de afastamento da máxima entropia. A unidade é kilocalorias ou joules.

A emergia define-se como a medida que expressa em energia o trabalho efetivo para realizar um produto, serviço ou processo natural (Odum, 1996). A emergia é uma medida que aponta para trás no tempo para registrar que quantidade de energia foi consumida no conjunto dos processos de transformações para realizar um trabalho qualquer. A emergia expressa a magnitude de um trabalho realizado em uma atividade produtiva, para um único produto, serviço e/ou processo natural. A unidade é emjoules ou em J.

Reportando-se a emergia de qualquer sistema à emergia solar tem-se que é a parte de energia solar utilizada, direta ou indiretamente, para realizar um serviço ou produto. A unidade é $(\mathrm{ems})=$ emergia solar em joule. (Odum, op. cit.)

A transformidade é a relação dimensional que expressa a taxa do fluxo de emergia solar usada para realizar um joule de qualquer trabalho (produto, serviço e/ou processo natural). A unidade é (solar emJ/Joule), ou $(\mathrm{ems} / \mathrm{J})$ ou ainda $(\mathrm{emj} / \mathrm{J})$. Quanto maiores as transformações energéticas para produzir um produto qualquer, maior é a transformidade. Isto ocorre porque a cada transformação, a energia disponível é usada para

\begin{tabular}{|c|c|}
\hline $\begin{array}{l}\text { EXERGIA / ENERGIA } \\
\text { TÍPICAS }\end{array}$ & PROCESSOS E CONDIÇÕES DETERMINANTES \\
\hline $\begin{array}{l}\text { terreno, cobertura vegetal, } \\
\text { declividade e atributos físicos }\end{array}$ & $\begin{array}{l}\text { o relevo atua como um potencial; as diversas condições restritivas } \\
\text { são vegetacão, tipo de solos, atributos físicos dos solos e rochas, } \\
\text { declividade e forma da bacia. }\end{array}$ \\
\hline $\begin{array}{l}\text { a chuva deverá ser } \\
\text { considerada com os produtos } \\
\text { químicos }\end{array}$ & $\begin{array}{l}\text { a chuva só tem exergia química se for portadora de substâncias, } \\
\text { especialmente reagentes, como ácidos e particulados. }\end{array}$ \\
\hline $\begin{array}{l}\text { a função produtividade } \\
\text { primária - transformação da } \\
\text { matéria orgânica em húmus }\end{array}$ & $\begin{array}{l}\text { a produtividade primária indica a disponibilização do folhedo, à ação } \\
\text { de micro- e macro- organismos, às alterações bioquímicas, à } \\
\text { disponibilidade de C, às diferenças de produtividade de distintas } \\
\text { assembléias vegetais, e as relações com a água / umidade. }\end{array}$ \\
\hline $\begin{array}{l}\text { formas das sub-bacias do } \\
\text { acumulação e produção de } \\
\text { escoamento superficial }\end{array}$ & $\begin{array}{l}\text { escoamento superficial, infiltracão, evaporacãa e evapotranspiracãa, } \\
\text { bem como a possibilidade de cheias em uma sub-bacia são } \\
\text { indicadoras de estilos de trocas dinâmicas com alta energia. }\end{array}$ \\
\hline $\begin{array}{l}\text { rochas como geradoras de } \\
\text { solos nas diversas condições } \\
\text { de trocas em relação às suas } \\
\text { condições de gênese }\end{array}$ & $\begin{array}{l}\text { rochas melanocráticas são as de maior exergia potencial; solos } \\
\text { oriundos desses tipos de rochas devem possuir mais nutrientes; } \\
\text { rochas leucocráticas seguem em importância de solos e rochas } \\
\text { sedimentares são as menos produtoras relativamente. }\end{array}$ \\
\hline $\begin{array}{l}\text { corpos de águas dentro de } \\
\text { lagos, represas, lagunas e } \\
\text { pântanos }\end{array}$ & $\begin{array}{l}\text { estratificacão de corpos d'água / diferenciação de corpos pelos } \\
\text { atributos físicos \{transportados, temperatura, redox, fauna, flora\} }\end{array}$ \\
\hline $\begin{array}{l}\text { clima como potenciais para a } \\
\text { erosão e incêndios naturais }\end{array}$ & $\begin{array}{l}\text { propensão a incêndios naturais / pluviosidade intensa / secas / secas } \\
\text { intermitentes / alta umidade / baixa umidade }\end{array}$ \\
\hline $\begin{array}{l}\text { propensão à perda universal } \\
\text { de solos }\end{array}$ & $\begin{array}{l}\text { a perda de solos pode ser produzida a um ritmo de igual reposição } \\
\text { natural; pode ser perda de massa estrutural ou lixiviação de } \\
\text { nutrientes sem alteração significativa da estrutura; pode ser perda } \\
\text { maior do que a produção natural. }\end{array}$ \\
\hline energia cinética dos ventos & transporte de sedimentos / erosão eólica \\
\hline energia cinética da chuva & chuvas catastróficas \\
\hline
\end{tabular}

Tabela 2: Alguns sistemas e transformações que podem ser expressas em exergia e emergia.

Table 2: Some systems and transformations that can be expressed in exergy and emergy. 
produzir uma menor quantidade de energia de outra forma. Assim a emergia cresce, a exergia decresce fortemente e a entropia aumenta (Odum, op. cit.).

Assim emergia, exergia, entropia, energia livre, energia interna e transformidade são as variáveis por excelência próprias para os estudos transdisciplinares aplicáveis à análise de sistemas naturais e culturais.

\section{REFERÊNCIAS}

AYRES, R.U., AYRES, L.W., MARTINAS, K. Ecothermadynamics: Exergy and Life Cycle Analysis. INSEAD. Working Paper Series. 1996. Fontainebleau, France.

GEORGESCU-ROEGEN, N. Energy and Economic Myths. New York: Pergamon Press. 1976

HUANG,Sh.L., FERNG,J.J.(1990 a) Applied Land Classification for Surface Water Quality Management: I Watershed Classification. Journal of Environmental Management. 31. p: 107-121.
HUANG,Sh.L., FERNG,J.J. (1990 b) Applied Land Classification for Surface Water Quality Management: II Land Process Classification. Journal of Environmental Management. 31. p: $127-141$.

JÖRGENSEN, S.E. Exergy and Ecology. Ecological Modelling. n.63. 1992. p. 185-214.

MARTINS Jr., P.P., ROSA,S.A.G., CÉSAR,F.M. (1994) Zoneamento em Áreas Homogêneas da Alta Bacia do Rio das Velhas com Base nas Características dos Geo-sistemas. VI SIMP. QUANTIFICAÇÃO GEOCIÊNCIAS. ANAIS VI. Rio Claro. p.

MARTINS Jr., P.P. A Abordagem Interdisciplinar e Seus Fundamentos. Belo Horizonte: Fundação CETEC. Projeto em Elaboração. 1995-1998.

ODUM, H. Environmental Accounting. Emergy and Environmental Decision Making. New York: John wiley \& Sons. 1996

ROSA,S.A.G., Martins Jr., P.P. Das Variáveis Significativas para um Geoprocessamento Cartográfico de Bacias Hidrográficas. Conceitos de Produtos Aplicativos Funcionais. Gramado: $X$ CONGR. BRAS. REC. HIDRICOS. 1993. 